\title{
LA TRADUCCIÓN LITERARIA TIENE LA PALABRA
}

\section{LITERARY TRANSLATION: THE FLOOR IS YOURS!}

\author{
Mabel Richart-Marset \\ Universitat de València
}

\section{ABSTRACT}

Taking as our starting point the moving necrology that literary translator Peter Bush dedicates to his professional colleague, Helen Lane, we delve into several key issues of literary translation: translation as «the circulatory system of the world's literatures» (Sontag, 2007: 17), the figure of the translator as a writer and his or her fruitful collaboration with the author when possible, his or her unwanted and forced invisibility in the works they translate, which has given rise to awareness campaigns, platforms of demands indeed, such as \#namethetranslator and \#TranslatorOnTheCover, the consumption of translated literature, etc. At the same time, a reflection is carried out on the status quo of the discipline of Translation Studies: Is there really a shift from Translation Studies to Translator Studies? Are we entering or have we already entered the era of Post-Translation Studies?

Key Words: Literary translation, Rewriting, Peter Bush, Translation studies, PostTranslation studies, \#TranslatorOnTheCover 


\section{RESUMEN}

Tomando como punto de partida la emotiva necrológica que el traductor literario Peter Bush le dedica a su colega de profesión, Helen Lane, nos adentramos en varios puntos clave de la traducción literaria: la traducción como «el sistema circulatorio de las literaturas del mundo» (Sontag, 2007: 17), la figura del traductor y de la traductora como escritores y su fructífera colaboración con el autor o autora cuando esta sea posible, su indeseada y forzada invisibilidad en las obras que traducen y que ha originado campañas de concienciación reivindicativas de la talla de \#namethetranslator y \#TranslatorOnTheCover, el consumo de literatura traducida, etc. Al mismo tiempo se lleva a cabo una reflexión sobre el statu quo de la disciplina de los Estudios de Traducción: ¿Se está realmente produciendo un giro desde los Estudios de Traducción hacia los Estudios del Traductor? ¿Estamos entrando o hemos entrado ya en la era de los Estudios de Post-Traducción?

Palabras Clave: Traducción literaria, Reescritura, Peter Bush, Estudios de Traducción, Estudios de Post-Traducción, \#TranslatorOnTheCover

Fecha de recepción: 12 de noviembre de 2021.

Fecha de aceptación: 7 de diciembre de 2021.

Cómo citar: Richart Marset, Mabel (2021): «La traducción literaria tiene la palabra», en Actio Nova: Revista de Teoría de la Literatura y Literatura Comparada, monográfico 5: 191-212.

DOI: https://doi.org/10.15366/actionova2021.m5.009 
«Translation is the circulatory system of the world's literatures》 (Susan Sontag, 2007: 177) «Translation asserts the possibility of a coherent, unified experience of literature in the world's multiplicity of languages) (Edith Grossman, 2010: 17) «Je est un autre, said the translator. Try next doon» (George Szirtes, 2019: 577)

\section{INTRODUCCIÓN}

El 15 de septiembre de 2004 en el periódico El País, se publicaba la necrológica de una de las más prolíficas y reconocidas traductoras literarias del siglo XX, la estadounidense Helen Lane, traductora de obras capitales de las literaturas española, francesa, italiana y portuguesa al inglés. Firmaba esta necrológica el también renombrado traductor literario británico, Peter Bush. Este último es muy conocido en nuestro país por traducir tanto del español como del catalán a la lengua inglesa, y en su haber cuenta con traducciones tan entrañables como La plaça del diamant de Mercè Rodoreda o L'últim patriarca de Najat El Hachmi. Ha recibido también numerosos premios de traducción literaria, entre los que se encuentran el prestigioso Ramón Llull (2014) por su traducción al inglés del Quadern gris de Josep Pla y el Valle Inclán (2012) por su traducción de la novela de Juan Goytisolo, El exiliado de aqui y de allá.

Es importante para nuestra investigación, recuperar aquí, varios fragmentos de tan emotiva despedida de Bush a su colega de profesión, porque entroncan directamente con varios de los puntos que nos gustaría abordar en este artículo: 


\section{NECROLÓGICA ${ }^{1}$ : Helen Lane, Traductora literaria.}

La traductora literaria Helen Lane falleció el 29 de agosto en Alburquerque, Nuevo México, a la edad de 83 años [...]. En el mundo anglosajón, tan reacio a la literatura traducida, Helen Lane consiguió forjarse un renombre insólito por sus traducciones de autores como Juan Goytisolo, Octavio Paz y Mario Vargas Llosa, y contribuyó enormemente a crear un lectorado para sus autores: raras veces faltaba su nombre en la portada de los libros, todo un símbolo de exactitud y belleza de la palabra traducida. Como comentario sobre toda su obra, vale la pena citar lo que dijo la Publishers Weekly acerca de su traducción de Yo, El Supremo, de Roa Bastos: "El lenguaje es un triunfo tanto para la traductora como para el autor: espléndidamente imaginativo, vitriólicamente brillante y vituperativo, rabelasiano en sus extravagancias".

Helen Lane dominaba siete idiomas, pero prefería trabajar con el francés, el italiano, el portugués y el español: a lo largo de su vida tradujo más de noventa obras [...]. Desde 1970 trabajó por libre y se instaló en una granja en la Provenza, donde le encantaba recibir a sus autores y hablar hasta bien entrada la noche de los retos que sus textos le planteaban $[\ldots]$.

Helen Lane era una mujer a la vez modesta y combativa en relación con su arte. [...] era capaz de llevar a juicio a las editoriales que querían cambiar un título que ella habia elegido por uno más blando, de acuerdo con la opinión de un departamento de ventas más preocupado por la publicidad.

[...] El primer premio que recibió fue por la traducción de El manifiesto surrealista de André Breton, y luego se le otorgarían el National Book Award (Corriente alterna, de Octavio Paz), el US Pen Award for Translation (El conde Julián, de Juan Goytisolo). En el 2000 fue galardonada con el Lifetime Achievement Award, el premio que concede la Asociación de Traductores literarios Americanos.

El primer punto que nos llama la atención del texto de Peter Bush es el referido a la resistencia del mundo anglosajón a la literatura traducida: «En el mundo anglosajón, tan reacio

\footnotetext{
${ }^{1}$ La cursiva de los fragmentos de la necrológica firmada por Peter Bush es nuestra. Se puede consultar la necrológica completa en la siguiente dirección electrónica: http://elpais.com/diario/2004/09/15/agenda/1095199211_850215.html
} 
a la literatura traducida, Helen Lane consiguió forjarse un renombre insólito por sus traducciones [...]». Y nos llama la atención por el marcado contraste que se produce con los países de habla no inglesa, donde el consumo de literatura traducida es muy elevado, a la par que muy valorado, como instrumento necesario para el acceso a otras culturas.

En España, por ejemplo, el consumo de literatura traducida, sobre todo de literatura en lengua inglesa, arroja unas cifras contundentes como lo refleja el estudio titulado La traducción editorial en España llevado a cabo por el Ministerio de Cultura español en 2008. Dicho estudio pone de manifiesto la relevancia de la traducción en este país donde se producen más de 20.000 títulos de obras traducidas al año, lo cual supone cerca de un $27 \%$ del total de la producción española de libros, situando a España entre los principales países traductores del mundo. El inglés mantiene el liderazgo como lengua de origen en las obras traducidas, representando el 50\% aproximadamente del total de las traducciones:

Este porcentaje nos sitúa entre los principales paises traductores del mundo, aunque hay que tener en cuenta que, a diferencia de lo que ocurre en otros países, en España se realiza un importante y cada vez mayor número de traducciones entre lenguas españolas: en el año 2007 supusieron el 24,3\% del total de traducciones y el 6,5\% del total de la producción editorial [...] Sobre el total de la obra traducida, se mantiene el tradicional liderazgo del inglés como lengua de origen, fluctuando en torno al $50 \%$ del total de traducciones. Concretamente, en el año 2007 las traducciones del inglés alcanzan el 47,5\% del total, algo por debajo de las cifras de 2006 (49,4\%). El mayor número de obras traducidas del inglés se produce en 1994, con un 55,4\% del total de traducciones. (Ministerio de Cultura de España, 2008: 8) (La cursiva es nuestra).

El segundo punto que contrasta, pero esta vez de forma positiva, con la situación actual del colectivo traductor a nivel global, es el relacionado con la invisibilidad del traductor o traductora: «Helen Lane consiguió forjarse un renombre insólito por sus traducciones [...] raras veces faltaba su nombre en la portada de los libros, todo un símbolo de exactitud y belleza de la palabra traducida $[\ldots] \gg$. Decimos que contrasta positivamente porque en la actualidad, los y las profesionales del sector de la traducción tanto a nivel nacional como internacional, siguen reclamando una visibilidad y unos derechos que a día de hoy se les siguen negando en numerosas ocasiones. Y es que desde que en 1995, el traductor y teórico de la traducción estadounidense, Lawrence Venuti (2018: 1), utilizara en su ya clásica obra, The Translator's Invisibility, el término «invisibilidad» para denunciar la situación y el trabajo de los traductores en las culturas tanto británica como estadounidense: «"Invisibility” is the term I will use to describe the translator's situation and activity in contemporary British and American cultures», muy pocas cosas han cambiado desgraciadamente en este sentido. 
Bush se muestra muy contundente a la vez que irónico en relación a esta cuestión cuando afirma que:

[...] La mayoría de los lectores de traducciones prefieren no saberlo, nos dicen la mayoría de los editores, que prefieren mantener al traductor oculto [...]. O tal vez, haya que aceptar sencillamente la muerte del traductor, su nombre adornando la solapa trasera como un sombrío epitafio a los meses de trabajo [...]. Al fin y al cabo, se trata de una metamorfosis que otorga al autor extranjero la condición de escritor inglés honorario, sin necesidad de pasaporte [...] (Bush, 2008: 23) (La cursiva y la traducción son nuestras).

Es rotundamente falsa la preferencia de los lectores por el anonimato de la figura de la traductora o traductor; y hay un sinfín de evidencia al respecto que lo demuestra. Por citar algunos ejemplos: ya en la década de los ochenta, el Comité de Traducción del PEN American Center, creó un «subcomité de vigilancia» que escribía cartas a los editores, directores de revistas y críticos de libros que omitían el nombre de los traductores literarios (Keeley, 1990: 294) para denunciar la ocultación. También dan testimonio de la escasa visibilidad del colectivo traductor a nivel mundial, las campañas \#namethetranslator, lanzada por las traductoras literarias Helen Wang y Louise Rogers Lalaurie en la red social de Twitter en 2013, y la nacida hace apenas unos meses, en septiembre de 2021, \#TranslatorsOnTheCover, capitaneada por el escritor Mark Haddon y la también escritora y traductora literaria Jennifer Croft. Estas campañas están aportando una nueva dimensión a la defensa de este colectivo.

Y aunque Wright asegure que las traductoras y los traductores están adquiriendo una mayor visibilidad, especialmente en Reino Unido, donde participan en calidad de expertos literarios en eventos de la talla de «Live Translation» $\mathrm{y}$ «World Literature», nosotros insistimos en que a la industria editorial todavía le queda mucho trabajo por hacer en su tarea de concienciación del justo reconocimiento al colectivo traductor como fuerza esencial creadora. De hecho la propia Wright reconoce, unas líneas más abajo, que «they (translators) are generally given credit for their work in newspapers and journals and are articulate in their protest when they are not»:

No cabe duda de que los traductores se han hecho más visibles. En el Reino Unido, por ejemplo, participan en lecturas, mesas redondas y otros actos en calidad de expertos literarios (los eventos Live Translation y World Literature de la London Review Bookshop son un ejemplo destacado de ello); se dirigen al público escolar a través del proyecto Translators in Schools, en el que trabajan con la riqueza cultural de una población diversa; generalmente se les reconoce su trabajo en periódicos y revistas y 
se manifiestan su protesta cuando no es así. (Wright, 2016: 3) (La cursiva y la traducción son nuestras).

El tercer y último aspecto que nos gustaría rescatar de la necrológica firmada por Bush es el referido a la estrecha colaboración que a veces se da entre escritora o escritor y traductora o traductor: «Desde 1970 trabajó por libre y se instaló en una granja en la Provenza, donde le encantaba recibir a sus autores y hablar hasta bien entrada la noche de los retos que sus textos le planteaban $[. ..] \gg$ o un poco antes, cuando se hace eco de una cita de la Publishers Weekly acerca de la traducción que hace Lane de la novela Yo, el Supremo del escritor paraguayo Roa Bastos: «El lenguaje es un triunfo tanto para la traductora como para el autor $[. .]$.$» .$

Qué duda cabe que cuando esta colaboración entre el tándem escritora-escritor y traductora-traductor tiene lugar, el resultado es óptimo. Bush asegura que él acostumbra a enviarles a los autores y autoras a quienes traduce, el borrador final de su traducción. En el ejemplo que nos relata a continuación, su traducción de la novela de Juan Goytisolo, Carajicomedia, publicada por Seix Barral en 2000, nos da cuenta de la fluida comunicación que mantuvo no sólo con el escritor español, que siempre, nos dice, respondía a sus consultas con detallados comentarios, sino también con el editor y el corrector de la editorial. Nos llama también mucho la atención, como a instancias del propio Goytisolo, Bush se puso en contacto con el traductor de la edición francesa para intercambiar sus respectivos borradores finales y ambos decidieron tras la revisión de dichos documentos, hacer cambios en sus respectivas traducciones:

\footnotetext{
I normally send my final draft translation to the authors that I translate. Juan Goytisolo always sends detailed comments and is ever prepared to respond to queries. At the suggestion of the author, the French translator, Claude Bleton and I, exchanged final drafts and made a few changes as a result. I have also received and responded to comments from the author, the publisher, Pete Ayrton of Serpents Tail, and the copy-editor, Ruth Petrie. One feature of this translation is that the author has changed the Spanish text from the one that was first published and that these changes will first see the light of day in the French and English translations. (Bush, 2008: 28) (La cursiva es nuestra).
}

Por consiguiente, la versión final que el lector o lectora recibe cuando lee una obra traducida, es el fruto de una serie continuada de esfuerzos por parte del traductor y del resto de agentes implicados en el proceso de traducción: «El que defineix l'acte de la traducció literària és l'esforç per donar una versió final que es publicarà. Aquest moviment cap a les paraules d'una página comporta un seguit de lectures, recerques, revisions i possibles col-laboracions amb l'autor i l'editor [...]». (Bush, 2003: 121). Sin embargo, el traductor británico reconoce que a pesar de todos 
estos esfuerzos en el proceso de traducción y por muy seguro o segura que el traductor o traductora pueda estar con su reescritura, cualquier acto de traducción está marcado por los «vaivenes de la inspiración» y por «las propias dudas», y que solo los lectores y lectoras pueden validar la traducción final impresa:

Naturally, any sustained act of translation is punctuated by ebbs and flows of inspiration, by self-doubt and scepticism and the final work in print can only be validated by its readers, however much the translator has researched, refined and is confident in what he or she has written. (Bush, 2008: 32) (La cursiva es nuestra).

También el nobel de literatura portugués José Saramago, autor traducido a más de 30 lenguas, señalaba en el encuentro internacional Saramago y sus traductores ${ }^{2}$, la necesaria e imprescindible labor de los traductores a los que otorgaba el poder de «aprender a leerse con ellos» y de convertir las literaturas nacionales creadas por los escritores de cada país en una «literatura universal»:

Los escritores hacen las literaturas nacionales y los traductores hacen la literatura universal: nos permiten a los que no podemos conocer todas las lenguas que se pueda leer algo escrito en Japón, Rusia, Finlandia... Los traductores convierten las lenguas en mi propia lengua; por eso seríamos más pobres sin ellos. Yo he aprendido muchisimo con ellos, he aprendido a leerme, porque a veces se plantean dudas que no sé aclarar. Todo está claro para mí en lo que escribo, pero no en lo que significa, y tengo que pensarlo varias veces. Ellos me han dicho que lo que uno escribe en su lengua no es tan fácil de entender. https://elpais.com/diario/1999/11/09/cultura/942102005_850215.html (La cursiva es nuestra)

Pero, ¿Cuál sería la definición que más se ajusta a lo que realmente es el proceso creativo de la traducción literaria? ¿En qué consiste realmente la labor de la traductora literaria y el traductor literario? ¿Qué lugar ocupa la Traducción literaria dentro de la disciplina de los Estudios de Traducción? ¿Se está realmente produciendo un giro desde los Estudios de Traducción hacia los Estudios del Traductor? ¿Estamos entrando o hemos entrado ya en la era de los Estudios de Post-Traducción? Trataremos de responder a estas preguntas a lo largo de este artículo.

\footnotetext{
${ }^{2}$ El encuentro internacional Saramago y sus traductores se celebró en 1999 en la Escuela de Traductores de Toledo, sede que alberga en la actualidad el observatorio permanente sobre traducción de lenguas mediterráneas. Se puede consultar el artículo completo en la siguiente dirección electrónica: https://elpais.com/diario/1999/11/09/cultura/942102005_850215.html
} 


\section{LA TRADUCCIÓN LITERARIA}

La traducción literaria es considerada como uno de los actos centrales de la comunicación humana y «por la diversidad de lenguas, una actividad imprescindible para la comunicación literaria» (Albaladejo, 1992: 94). Recuperamos también ahora la cita de Susan Sontag con la que dábamos comienzo a este artículo: «Translation is the circulatory system of the world's literatures» (2007: 177) (La cursiva es nuestra).

De la misma manera que el sistema circulatorio del ser humano, con el corazón y sus casi 100.000 kilómetros de vasos sanguíneos en continuo viaje es vital para nuestra supervivencia, la literatura traducida es el único vehículo del que disponemos que nos permite realizar «un viaje mental» a cualquier época y lugar del mundo; nos asegura la circulación de los textos literarios a través de las fronteras temporales y culturales, por lo que se vuelve vital para nuestra supervivencia como sociedad cuidar de tan preciado instrumento que nos posibilita y asegura el contacto con la diversidad de culturas que conforman nuestra aldea global. De hecho, dar la vuelta al mundo bordeando la línea del Ecuador, implica llevar a cabo un recorrido de unos $40.000 \mathrm{kms}$, viaje que podríamos realizar infinidad de veces en nuestra vida de la mano de la traducción literaria:

My sense of what literature can be, my reverence for the practice of literature as a vocation, and my identification of the writer with the exercise of freedom-all these constituent elements of my sensibility are inconceivable without the books I read in translation from an early age. Literature was mental travel. Travel into the past and to other countries. (Sontag, 2007: 179). (La cursiva es nuestra).

Bush (2003: 121-123) parte del supuesto de que la traducción literaria es «una forma de escritura única y distintiva», y en consecuencia, la traductora o traductor literario tiene que ser y convertirse en «a unique, distinctive form of writer». Define la traducción literaria como una práctica artística que tiene lugar en un contexto comercial socioeconómico: «The specificity of the practice as a complex subjective process within defined historical, socio-economic contexts of human communication requires detailed studies of that material process». Ponemos el énfasis en dicho contexto comercial socio-económico porque es la base para 
poder entender que en última instancia el fin de la traducción literaria como cualquier otro producto creado en una sociedad de consumo, es «ser vendido» ${ }^{3}$ :

\begin{abstract}
A literary translation is an artistic practice which takes place in a commercial socioeconomic context. The writer wants his work to be read in other languages and cultures, the publisher hopes this will happen, that books will be sold [...] A living writer, his publisher, a copy-editor may well intervene in the process of drafting [...] There is no pure, individual agency for the writing as the translator must respond to the various collaborations offered: though changes that ensue can be moulded by the translator's over-all feel for the text, sometimes the climate can become more litigious, writers and editors can insist on what the translator finds unacceptable and the latter has the right to remove bis name from the translation. This crucial area in the negotiation of texts is little referred to in Translation Studies. (Bush, 2003: 123) (La cursiva es nuestra).
\end{abstract}

Hay otros dos aspectos importantes que merecen ser destacados de esta última cita de Bush. El primero de ellos es el referido a la «agency» de la traducción literaria. Bush (2003:123) asegura que el agente traductor no es el único agente creador de la versión final de la traducción («There is no pure, individual agency for the writing») porque debe responder a las distintas colaboraciones propuestas por los otros agentes que forman parten del proceso creativo de la traducción literaria, ya sea el propio autor o autora del texto literario que va a ser traducido (en el caso, claro está de que estos últimos estén vivos), el editor o editora del sello editorial que publicará el texto traducido, el corrector o correctora de textos, etc.

En la misma línea, Susan Bassnett en el prólogo del libro Translation and Rewriting in the Age of Post-Translation Studies (Gentler, 2017) señala el creciente interés en estudiar la traducción como una «actividad plurivocal» donde junto con las voces del traductortraductora y autor-autora se combinan muchas otras no mencionadas: «[...] we can add the growing interest in seeing translation as a plurivocal activity, since many other voices than those of translator and 'original' author combine in the actual translation process». (Bassnett, 2017: ix)

Nos parece cuanto menos curioso el paralelismo que de nuevo se produce entre los procesos creativos de la traducción literaria y los de la traducción audiovisual (TAV). En la obra citada anteriormente (Richart-Marset: 2012), señalábamos también la existencia de una caja negra del doblaje que contiene los documentos que dan fe de las distintas

\footnotetext{
${ }^{3}$ En este aspecto, la traducción literaria no se diferencia demasiado de otro tipo de traducciones. Por ejemplo, en Ideología y traducción. Por un análisis genético del doblaje, Richart-Marset define el doblaje dentro de la traducción audiovisual, destacando su faceta de expansión comercial: «es un proceso de expansión cultural y comercial consistente en la traducción, moldeamiento y transformación de la dimensión verbal de un filme [...] en cuya realización intervienen diferentes agentes». (Richart-Marset, 2012: 14) (la cursiva es nuestra).
} 
manipulaciones-transformaciones llevadas a cabo por los distintos agentes que participan en la cadena (agente ajustador, agente de dirección de doblaje, actrices y actores de doblaje, etc).

Las voces de estos agentes, al igual que sucede en la traducción literaria, tampoco se mencionan, se ocultan en esa caja negra continente de un archivo secreto que, por razones de política empresarial u otras que no alcanzamos a desvelar, se guarda bajo llave y se impide el acceso democrático:

[...] existe también una caja negra, poco mencionada en los estudios sobre el tema, a veces entrevista y, desde luego, muy pocas veces exhibida. [...] En el caso del doblaje dicha caja tiene una existencia documental, textual, testimonial, que por razones de política empresarial, u otras, se oculta, se guarda bajo secreto y se impide el acceso democrático. Dicho de otro modo: la caja negra del doblaje es un archivo oculto a la mirada del especialista y del público en general. (Richart-Marset, 2012: 8) (La cursiva es nuestra).

\section{CRÍtTICA A LOS ESTUdIOS DE TRADUCCIÓN}

El segundo aspecto que queremos recuperar de la cita de Bush del apartado anterior (2003: 123), está enfatizado en las últimas líneas. En ellas el traductor británico acusa a los Translation Studies de no dar cuenta de lo que realmente sucede en el ejercicio práctico de la traducción cuando, por ejemplo, no se llega al consenso en la versión final traducida entre el triángulo formado por escritor-a/traductor-a/editor-a.

Bush considera que esta es un área crucial de negociación ya que, en caso de no llegar a un acuerdo, la traductora o traductor tiene el derecho de retirar su nombre de la traducción: «sometimes the climate can become more litigious, writers and editors can insist on what the translator finds unacceptable and the latter has the right to remove his name from the translation. This crucial area in the negotiation of texts is little referred to in Translation Studiess.

El traductor británico denuncia públicamente a los Estudios de Traducción que, en su empeño de querer instalarse a toda costa en las universidades, adolecen de una falta de interdisciplinariedad necesaria para dar cuenta del proceso creativo que es la traducción literaria. Esta falta de cooperación con otras disciplinas desembocará inevitablemente en una visión limitada del fenómeno traductológico (Bush, 2003: 122): «In its struggle to establish itself within universities, Translation Studies has adapted to or become enmeshed in various subject power struggles that threaten what should be an essential interdisciplinarity with tunnel vision». 
Se queja profundamente de la ingente cantidad de libros y artículos integrados en una genealogía autorreferencial limitada a los Estudios de Traducción, cuando la mayor parte de esa investigación debe sus teorías críticas a disciplinas anteriores (Bush, 2003:122): «legion are the articles and books embedded in self-referential genealogies limited to Translation Studies when most of the individuals concerned in fact owe their critical theories to pre-existing disciplines». Bush mantiene que dichas teorías se suceden manteniendo una espinosa relación con la práctica y cuando él mismo se pregunta el porqué de este proceder, lo tiene muy claro:

Theories are pursued in an often relationship with practice. Why? Because university ideals of scholarship privilege a disconnection from 'doing it' in a tradition of scientific objectivity that belittles the practising subject, or of an education that flees contamination with the vocational.(Bush, 2003:122) (La cursiva es nuestra).

Como acabamos de ver, Bush achaca esa falta de conexión entre teoría y práctica a los ideales de erudición de la universidad, que privilegian claramente la desconexión del «hacer», en su más pura tradición de objetividad científica que menosprecia la práctica. Va incluso un poco más allá cuando afirma que detrás de todas estas cuestiones subyacen otras verdades más polémicas, entre las que incluye, entre otras, un claro interés de supervivencia profesional por parte de los académicos que abrazaron los Estudios de Traducción después del ocaso de las enseñanzas de lenguas extranjeras y la literatura comparada, y la consagración de los debates teóricos y prácticas de investigación que confunden la naturaleza esencial de escritura de la traducción en el seno de las humanidades:

Behind these issues lurk more controversial realities: academic staff who have embraced Translation Studies for professional survival after the collapse of foreign language or comparative literature teaching, [...] and the enshrining of theoretical debates and research practices that obfuscate the essentially writerly nature of translation within the Humanities[...]. (Bush, 2003: 122) (La cursiva es nuestra).

Especial atención nos merece al mismo tiempo que nos preocupa, la reflexión que hace Bush sobre los estudiantes de traducción literaria, pues considera que estos últimos se encuentran inmersos en una maraña de estrategias de traducción y tipos de análisis durante sus años de formación, sin detenerse a confrontar de manera crítica su capacidad para escribir de forma creativa como traductores, ya que se parte del supuesto de que la teoría «correcta» llevará a la práctica «correcta»: 
critically confront their ability to write creatively and imaginatively as translators because the assumption is created that the 'right' theory will lead to the 'right' practice. (Bush, 2003: 122) (La cursiva es nuestra).

Sin embargo, estas críticas no se detienen aquí, y siguen acentuándose con el paso de los años. En 2007, Bush edita junto con Susan Bassnett, el libro The Translator as Writters. En el capítulo que lleva su firma, «The writer of Translations», título por cierto que constituye toda una declaración de principios, ya no oculta su profundo malestar con la poca implicación de los Estudios de Traducción para con la traducción literaria, y acusa directamente a la mayoría de los especialistas académicos de la traducción, quienes en su afán de no querer ensuciar su «impoluta ciencia lingüística» con las experiencias «caóticas, fastidiosas y creativas» de los traductores literarios profesionales, silencian el hecho de que la traducción literaria constituye «el acto de escritura más valiente y osado»:

Silence is the best policy, it seems, when it comes to describing the act of literary translation as the boldest act of writing.[...] and most academic translation specialists, who ought be heralds of the art, prefer to keep their linguistic science unpolluted by the messy, inventive, irksome experiences of professional literary translators engaged in the adventure of multiple rereadings and rewritings of the carefully placed words of another writer in another language [...]. (Bush, 2007: 23) (La cursiva es nuestra).

Su posicionamiento a favor de describir con todo lujo de detalles la experiencia práctica de la traducción literaria, no deja lugar a dudas. Una práctica desafiante que es necesario sacar a la luz si se quiere describir la verdadera naturaleza de la traducción literaria como un proceso creativo, difícil y complejo de relecturas y reescrituras. Sólo así, desde la práctica, será posible reconocer «la traducción literaria como uno de los actos centrales de la comunicación humana» (Bush, 2007: 23): «However, as I prefer the mess of reality to secrets and silence and want literary translation to be recognized as one of the central acts of human communication, I will attempt a description of an experience of translation».

Por otro lado, Peter Constantine, también aboga por un cambio de dirección en los Estudios de Traducción. En su reciente estudio, «Professionalisation of Literary Translation and the Publishing Market» constata que se está produciendo un creciente interés hacia los estudios de la figura del traductor o traductora frente a los estudios de traducción: «there has also been a growing interest in translator studies as opposed to translation studies» (Constantine, 2019: 99). Declara que este nuevo campo de estudio no se enfocaría en la traducción sino en la propia figura del traductor o traductora desde una perspectiva 
sociológica, evaluando los procesos de toma de decisiones en todos los aspectos de su trabajo.

En la misma línea, Chesterman (2017: 16) sostiene que los estudios centrados en la figura del traductor o traductora, cubrirían facetas tales como el estatus de los distintos tipos de traductores en las diferentes culturas, las tarifas, las condiciones de trabajo, los sistemas de acreditación, las asociaciones profesionales, las redes de traductores, cuestiones como los derechos de autor, etc.

\section{LA TAREA DE LA TRADUCTORA O TRADUCTOR}

Mucho se ha escrito en torno a este tema desde que Walter Benjamin en su célebre ensayo, «La tarea del traductor», afirmara que la tarea de este último era «encontrar en la lengua a la que se traduce una actitud que pueda despertar en dicha lengua un eco del original» (1967: 83). Para Bush (2003: 122), por ejemplo, la traductora o el traductor literario: «works with a text written by someone else in another language which he transforms into writing in another language»; pero esta afirmación, en apariencia simplista, esconde un complejo proceso de transformación. Dicho proceso supone un largo camino de relecturas y reescrituras por parte del agente traductor, en un esfuerzo denodado cuyo fin es tratar de «comprender» a través de una lectura atenta (close reading, al más puro estilo de la crítica literaria) y «crear», como lo haría cualquier otro agente que lee y escribe literatura:

The translator reads, re-reads, writes, re-drafts, self-edits, re-writes. During this struggle to understand and create, like any other reader and writer of literature, the words will appeal to the translator's emotions, memories, ideas within an intense flow of subjective consciousness. This is not an operation in a void, not a clinical transplant of meaning but a process of literary reading and writing. (Bush, 2003: 122) (La cursiva es nuestra).

En este sentido el traductor o traductora se convierte en ese «lector implícito» (implizite Leser) del que hablaba el teórico de la literatura alemán Wolfgang Iser, que hace posible en su interacción a través de la lectura con el texto literario, que este último exista. En otras palabras, el texto solo podría existir en la medida que es leído, de modo que el sujeto que lee y el sujeto que escribe participarían en el mismo juego imaginario. 
La obra literaria existe desde el mismo instante en que el texto creado por una autora o autor entra en relación con la lectura concreta que hace su lectora o lector: «Las significaciones de los textos literarios sólo se generan en el proceso de lectura; constituyen el producto de una interacción entre texto y lector, y de ninguna manera una magnitud escondida en el texto». (citado por Manuel Asensi, Historia de la teoría de la literatura II, año 2003: 680). El lector implícito, por consiguiente, limita las posibles interpretaciones del texto literario, no es un lector real sino «la totalidad de la pre-orientación que un texto de ficción ofrece a sus posibles lectores», de ahí que «ninguna lectura pueda nunca agotar todo el potencial, pues cada lector concreto llenará los huecos a su modo» (citado por Manuel Asensi, Historia de la teoría de la literatura II, año 2003: 680-681).

Por lo tanto, esa lectura atenta por parte del agente traductor, siempre tendrá un componente marcadamente subjetivo, como no podría ser de otra manera:

El traductor no és un subjecte despersonalitzat, sinó una subjectivitat crítica, bistòrica $i$ emocional. Els diferents nivells de consciència estan fusionats per la recreació del text original, una arquitectura verbal i musical l'elaboració de la qual no és captada adequadament per la majoria dels conceptes actualment en boga en els Translation Studies. (Bush, 2003: 121) (La cursiva es nuestra).

No cabe duda de que esa lectura atenta por parte de la traductora o traductor está siendo transformada en la actualidad por la tecnología digital. Goicochea, Llamas, Sánchez y Sanz (2020:1) lo tienen claro: «Literatures born digital can show how rituals of reading, formulas of production, and narratives are being modified in the twenty-first century».

En la misma línea, el traductor estadounidense Edwin Gentler, reflexiona sobre los procesos de lectura y reescritura en la era actual de la reproducción digital (o reproducción electrónica como utilizan en el contexto anglosajón). Parafraseando a Walter Benjamin, Gentler traslada el concepto de «aura del original», el cual sufrió una drástica transformación en la era de la imprenta al generarse una reproducción masiva de libros, al «aura de la traducción», argumentando que esta última ha sufrido también un cambio notable en la era de la reproducción electrónica: «I argue, that the aura of translation has changed in the era of electronic reproduction» (Gentler, 2017:14).

$\mathrm{Y}$ es que los nuevos medios de comunicación han transformado profundamente tanto la manera de escribir, traducir y reescribir historias como la manera de «leer-navegar» el texto reescrito: «The new media not only alters how authors and translators write, translate, and rewrite stories, but also alters how readers and viewers navigate the rewritten text» 
(2017:14). Esto tiene una consecuencia directa para el lector y lectora, quienes a través de las herramientas que les ofrecen las nuevas tecnologías, como por ejemplo los hipervínculos que permiten leer los textos de manera no secuencial, pueden confeccionarse un modo de abordar el texto «a la carta», manipulando así el proceso de lectura.

Este nuevo modo de lectura provocaría un cambio de roles, en el que como apunta Gentler, el lector-receptor se convertiría en autor o mejor dicho en reescritor: «close-ups, cross- tracking, and links to tangential texts now allow readers to enter the text and to manipulate the reading process, turning receivers into authors or, better said, rewriters themselves). (2017:14) (La cursiva es nuestra).

Volviendo a la tarea de reescritura en la que como acabamos de ver, todo traductor o traductora participa, estamos totalmente de acuerdo con Gentler (2017:11) en que el hecho de romper el dominio de la adhesión servil al texto original siempre da lugar a mejores traducciones en muchos sentidos. Peter Rutherford, hispanista y traductor literario, responsable de una de las mejores traducciones de Don Quijote de la Mancha al inglés, constituye un claro ejemplo de lo que estamos diciendo cuando afirma, y le cito literalmente que: «Ahora que estoy siendo inmodesto, añadiré que algunos de mis chistes y poemas son mejores que los de Cervantes». (Rutherford, 2007: 79).

¿Está siendo Rutherford, realmente inmodesto, o por el contrario está en lo cierto cuando afirma que algunos de sus chistes y poemas son mejores que los de Cervantes? Vale la pena escucharle porque su argumentación encaja perfectamente en lo que venimos defendiendo hasta ahora en relación a la tarea del agente traductor como práctica de reescritura creativa:

Tal afirmación es una herejía para algunos, pero es perfectamente razonable: los traductores pueden mejorar el original, porque la lengua de llegada está obligada a ofrecer posibilidades expresivas no disponibles en la lengua de partida (el poder de síntesis del verbo inglés, por ejemplo); y también porque el primer escritor de una novela tiene que llevar su imaginación hasta el límite para crear tanto el mundo funcional como las frases con las que retratarlo, mientras que sus escritores posteriores, sus traductores, heredan el mundo funcional del primer escritor y pueden concentrar sus poderes imaginativos y creativos en las frases. Es mucho lo que se puede ganar, asi como lo que se puede perder en la traducción. Me parece aconsejable que los traductores mejoren siempre que puedan, para compensar los otros lugares en los que sus versiones deben ser menos efectivas que el original, porque la lengua de origen está obligada a ofrecer posibilidades expresivas no disponibles en la lengua de destino (la flexibilidad de la sintaxis española, por ejemplo). Y cuando mi versión de un soneto o de un chiste o de una frase resultaba mejor que la de Cervantes, ¿¿debia reescribirla para hacerla peor? (Rutherford, 2007: 79) (La cursiva y la traducción son nuestras). 
De lo que no nos cabe duda es que, para poder entender y valorar la tarea del traductor o traductora, es importante que los lectores de literatura traducida entiendan que la equivalencia completa entre dos lenguas es, como asegura Baer (2019: 59), una utopía, debido a que por un lado existe una asimetría entre las lenguas, (esas «posibilidades expresivas no disponibles en la lengua de partida o en la lengua de llegada» de las que habla Rutherford), y por otro lado, a que la naturaleza polisémica es frecuente en la mayoría de las palabras:

These two linguistic realities - that of the asymmetry of language and the polysemous nature of most words - support the idea of translation not as a linguistic matching game but as a complex decision-making process (see Levý 2000), in which one's overall interpretation of a text will affect translation decisions at every level and where translation decisions at the beginning of the text will shape all subsequent decisions. (Baer, 2019: 60).

Con esta concepción de la tarea de la traductora o traductor, continúa Baer (2019:60), los lectores ya no deben preguntarse si la toma de esta decisión u otra por parte del agente traductor está bien o mal, sino más bien si ha sido coherente o no: «With this understanding of the translator's task, readers can ask whether the translator's decisionmaking is informed, consistent or responsible rather than right or wrong».

Nos gustaría terminar este apartado con dos citas que engloban varias de las cuestiones tratadas aquí y con las que comulgamos totalmente. La primera cita pertenece a David Damrosch (2009: 66), quien afirma que: «an excellent translation can be seen as an expansive transformation of the original, a concrete manifestation of cultural exchange and a new stage in a work's life as it moves from its first home out into the world» ${ }^{4}$.

La segunda cita pertenece al libro, The Translator in the Text: On Reading Russian Literature in English de Rachel May (1994: 109): « if we persist in believing that a reader should not be able to tell she is reading a translation, then we devalue the translator's art and remove one of the main reasons to produce or read translations in the first place: the chance to glimpse another culture» ${ }^{5}$.

\footnotetext{
4 «Una traducción excelente puede considerarse aquella que constituye una transformación expansiva del original, una manifestación concreta del intercambio cultural y una nueva etapa en la vida de una obra que se desplaza desde su primer hogar hacia el mundo». (La traducción es nuestra)

${ }^{5}$ « Si nos empeñamos en creer que una lectora o lector no debería ser capaz de decir que está leyendo una traducción, entonces devaluamos el arte del agente traductor y eliminamos una de las principales razones para crear o leer traducciones en primer lugar: la oportunidad de vislumbrar otra cultura». (La traducción es nuestra)
} 
4. BREVE REFLEXIÓN FINAL SOBRE EL FUTURO DE LOS ESTUdiOS DE TRADUCCIÓN: LOS ESTUDIOS DE POST-TRADUCCIÓN

En la introducción de su libro, Translation and Rewriting in the Age of Post-Translation Studies, Edwin Gentler reflexiona sobre el statu quo de los Estudios de Traducción en la actualidad y sobre el surgimiento de un nuevo giro en el seno de estos últimos, giro que ha sido acuñado con el término de «Estudios de Post-Traducción», e introducido para intentar dar cabida a otros tipos de textos y objetos distintos a los analizados hasta ahora por la academia. Nos hacemos eco de sus palabras:

En el campo de los estudios de traducción, el término "estudios de post-traducción" se ha introducido para tratar de ampliar los tipos de textos y objetos que los académicos examinan. El nombre fue acuñado por Siri Nergaard y Stefano Arduini en "Translation: A New Paradigm" (2011), la introducción al primer número de una nueva revista llamada Translation, fundada en 2011. (Gentler, 2017: 1) (La cursiva y traducción son nuestras)

Nergaard y Arduini (2011: 8), acuñadores del término, proponen «the inauguration of a transdisciplinary research field»y describen una nueva era en la que la traducción es considerada principalmente como una materia «transdiciplinar, móvil y abierta». Estos abogan claramente por una investigación de la traducción desde otras disciplinas tales como el arte, la psicología, la filosofía, los estudios de género, los estudios étnicos, etc... :

Proponemos la inauguración de un campo de investigación transdisciplinar con la traducción como herramienta interpretativa y operativa. Imaginamos una especie de nueva era que podría denominarse estudios de postraducción, en la que la traducción se considera fundamentalmente transdisciplinar, móvily abierta [...] sugiriendo que el campo se abra a las investigaciones de la traducción desde fuera de la disciplina: desde el arte, la arquitectura, la etnografía, los estudios de la memoria, el paisaje, la psicología, la semiótica, la filosofía, la economía, los estudios de género, la raza, la clase y los estudios étnicos ([2011: 8] citado por Gentler, 2017: 1) (La cursiva y traducción son nuestras).

Y es que como asegura Gentler (2017:1), el campo de los Estudios de Traducción hasta ahora ha sido muy limitado, centrado en el texto («text-centric»), y basado en definiciones y modelos europeos derivados de los años 70 y principios de los 80 . El traductor y crítico de literatura comparada estadounidense, establece un claro paralelismo entre el fin de los Estudios de Traducción y la obra de la crítica literaria india, Gayatri Chakravorty Spivak, Death of a Discipline (2003). Este libro supuso también en su día un giro radical en el 
seno de los estudios de la literatura comparada al reclamar un «abanico más amplio de investigaciones disciplinarias, que incluyeran necesariamente los discursos de género, de las minorías y del Tercer Mundo junto con su traducción»:

In Death of a Discipline (2003), Gayatri Spivak discussed opening up the field of comparative literature, which she viewed as Eurocentric and based on outdated comparative literary studies. Instead she called for a broader array of disciplinary investigations, which necessarily included gender, minority, and Third World discourses and their translation. In a similar fashion, some scholars find the field of translation studies too narrow, text-centric, and based upon European definitions and models derived in the 1970s. (Gentler, 2017:1)

Gentler (2017:1), al igual que Spivak (2003), concluirá reclamando una investigación sobre los fenómenos de la traducción no inscrita en una única disciplina: «[...] los fenómenos de la traducción aparecen en todas las lenguas, en los discursos mayores y menores, y en muchas formas de comunicación, no sólo en los textos escritos» y haciendo una clara llamada a la acción: «Hay que dejar que estos elementos florezcan, informen e instruyan».

Nos gustaría concluir este artículo con una reflexión que hace Susan Bassnett (2017: ix-x) en el prólogo del libro de Gentler donde declara que los Estudios de Traducción no están moribundos y contempla los Estudios de Post-Traducción como un nuevo y apasionante giro en la disciplina, al que bautiza con el nombre de «Outward Turn». Concluye el prólogo, afirmando que los Estudios de Post-Traducción «pueden ser el catalizador para llevar la disciplina a una nueva etapa de desarrollo y posicionar la traducción como una condición cultural fundamental que subyace a la comunicación en el siglo XXI:

Translation studies is not moribund; post-translation studies is an exciting new phase for the subject. We have seen a great many 'turns' in translation studies; now is the time for the "Outward Turn" as the field redefines itself and begins to enjoy greater exchange with other disciplines in a mutually beneficial process of importing and exporting methodologies and ideas. Post-translation studies may annoy diehards with overly rigid views about studying translation, but it may well prove the catalyst for taking the subject forward into a whole new stage of development and positioning translation as a fundamental cultural condition underlying communication in the twenty-first century. (2017: ix-x) (La cursiva es nuestra). 


\section{BIBLIOGRAFÍA}

Albaladejo, Tomás (1992): «Aspectos pragmáticos y semánticos de la traducción del texto literario» en Koiné. Quaderni di ricerca e didattica sulla traduzione e l'interpretazione, anno Il, fascicoli 1-2: 179-200.

Asensi, Manuel (2003): Historia de la teoría de la literatura, vol. II, Valencia, Tirant lo Blanch.

Baer, Brian James (2019): «Teaching Literature in Translation» en Washbourne, Kelly; Ben Van Wyke (eds.) (2019): The Routledge Handbook of Literary Translation, New York, Routledge.

Bassnett, Susan; Peter Bush (eds.) (2007): The Translator as Writer, Bloomsbury Publishing.

Benjamin, Walter (1967): Ensayos escogidos, Buenos Aires, Sur.

Bush, Peter (2003): «The Act of Translation: The Case of Juan Goytisolo’s A Cock-Eyed Comedy», en Quaderns. Revista de traducció, 10: 121-134.

Bush, Peter (2007): «The writer of Translations» in Bassnett, Susan and Peter Bush (eds.) (2007): The Translator as Writer, Bloomsbury Publishing.

Constantine, Peter (2019): «Professionalisation of literary translation and the publishing market» in Washbourne, Kelly; Van Wyke, Ben (eds.) (2019): The Routledge Handbook of Literary Translation, New York, Routledge: 89-102.

Chesterman, Andrew (2017): «The Name and Nature of Translator Studies», in HERMES. Journal of Language and Communication in Business, 42: 13-22.

Damrosch, David (2009): How to Read World Literature. Malden, Oxford, Wiley Blackwell.

Gentler, Edwin (2017): Translation and Rewriting in the age of Post-Translation Studies, New York, Routledge.

Goicochea, María; Llamas Miriam; Sánchez Laura y Amelia Sanz (2020): «Digital Literatures Circulating in Spanish: The Emergence of a Field» en Bachleitner, Norbert (ed.) (2020): Literary Translation, Reception, and Transfer, De Gruyter: 385-395.

Keeley, Edmund (1990): «The Commerce of Translation» in Journal of Modern Greek Studies 8 (2): 291-297.

Levý, Jirí (2000): «Translation as a Decision Process» in Venuti, Lawrence (ed.): The Translation Studies Reader, London, Routledge: 148-159.

May, Rachel (1994): The Translator in the text: On reading Russian Literature in English, Northwestern University Press. 
Ministerio de Cultura, Centro de documentación del libro y la lectura (2008): La traducción editorial en España.

Nelson, Brian; Brigid Maher (eds.) (2013): Perspectives on Literature and Translation. Creation, Circulation, Reception, New York, Routledge.

Richart-Marset, Mabel (2012): Ideología y Traducción. Por un análisis genético del doblaje, Madrid, Biblioteca Nueva.

Rutherford, John (2007): «Translating fun: Don Quixote» in Bassnett, Susan and Peter Bush (eds.) (2007): The Translator as Writer, Bloomsbury Publishing.

Spivak, Gayatri (2003): Death of a discipline, New York, Columbia University Press.

Szirtes, George (2019): «Afterword. The death of the Translator» in Washbourne, Kelly; Ben Van Wyke (eds.) (2019): The Routledge Handbook of Literary Translation, New York, Routledge.

Venuti, Lawrence (2018, 1995): The Translator's Invisibility. A History of Translation, New York, Routledge.

Wright, Chantal (2016): Literary Translation, New York, Routledge. 


\section{SOBRE LAS AUTORAS}

\section{Mabel Richart-Marset}

MABEL RichART-MARSET es profesora de la Universitat de València desde el año $2006 \mathrm{y}$ profesora invitada en distintas universidades europeas y estadounidenses donde imparte seminarios y conferencias sobre estudios de cine y traducción. En el año 2017, fue nombrada Distinguished Visiting Professor por la University of Virginia. Doctora en Comunicación Audiovisual, sus principales áreas de investigación giran alrededor de los estudios fílmicos, la traducción audiovisual, los estudios de género y la enseñanza del español para extranjeros. Es autora de seis libros y numerosos artículos sobre estudios fílmicos, traducción, interpretación de conferencias y análisis del discurso. Entre sus libros destacan La alegría de transformar: teorías de la traducción y teoría del doblaje; Fraseología y traducción: una semiótica difusa e Ideología y Traducción. Por un análisis genético del doblaje.

MABEL RICHART-MARSET is professor at the University of Valencia and visiting professor at several European and American universities where she is invited to speak at seminars and lectures on translation and film. In 2017, she was named Distinguished Visiting Professor by the University of Virginia. She holds a $\mathrm{PhD}$ in Audiovisual Communication and her main areas of research revolve around film studies, audiovisual translation, gender studies and the teaching of Spanish as a foreign language. She is the author of six books and numerous articles on film studies, audiovisual translation, conference interpreting and discourse analysis. Her books include La alegría de transformar: teorías de la traducción y teoría del doblaje, Fraseología y traducción: una semiótica difusa and Ideología y Traducción. Por un análisis genético del doblaje. 